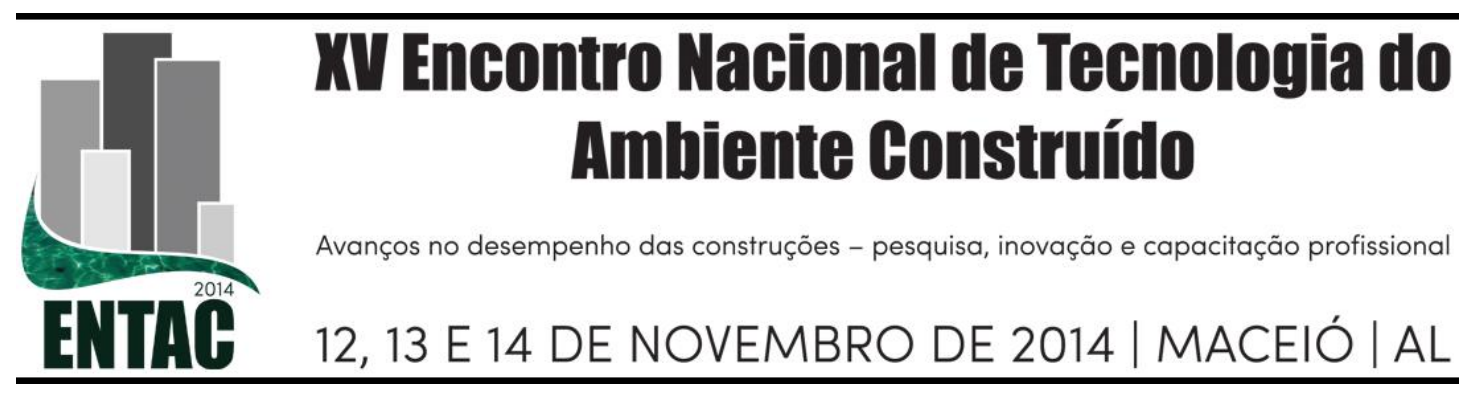

\title{
BIM: CONHECIMENTOS NECESSÁRIOS E DESAFIOS INICIAIS DE ADAPTAÇÃO
}

NASCIMENTO, Victor Hugo (1); CARDOSO, Carina (2); BORGES, Marcos (3)

(1) UFJF, e-mail: victorhugodoy@gmail.com (2)UFJF, e-mail: carinafolena@hotmail.com, (3) UFJF, marcos.borges@engenharia.ufjf.br

\begin{abstract}
RESUMO
A tecnologia BIM vem cada vez mais sendo requisitada e utilizada no mercado de AEC. O dinamismo do mercado e a complexidade cada vez maior dos produtos gerados fazem com que o uso de ferramentas computacionais compatíveis se torne cada vez mais importante. No entanto, acompanhar e compreender estes avanços tem sido um desafio para os profissionais usuários destes sistemas. Tendo isso em conta, muitas universidades criaram cursos para ensinar a tecnologia BIM aos alunos de Arquitetura e Engenharia, o que contribui para sua consolidação como importante ferramenta de projeto. Ainda assim, muitos alunos ainda preferem utilizar a tecnologia CAD convencional. O presente artigo faz um levantamento das principais dificuldades encontradas pelos alunos da disciplina de BIM do curso de Arquitetura e Urbanismo da UFJF e as razões que levam grande parte destes a não adotar a tecnologia BIM no processo de projeto. Trata-se de uma pesquisa explicativa que visa compreender as raízes das dificuldades no uso dessa ferramenta. Para tanto, num primeiro momento, foi realizada uma revisão bibliográfica a fim de se levantar os conhecimentos prévios necessários ao desenvolvimento de projetos nas plataformas BIM. Depois, os estudantes elegeram as maiores barreiras no uso dessa tecnologia. Os resultados mostram que a maior dificuldade encontrada por eles é a grande quantidade de dados que precisam ser inseridos no programa para poder projetar. Segundo os estudantes, isso torna o processo mais lento e complexo. Uma análise mais profunda revela, porém, que essa dificuldade deve-se principalmente à falta de conhecimento do próprio processo de construção, saber necessário à execução de projetos nas plataformas BIM. Conclui-se, daí, que é mais produtivo ensinar aos alunos a tecnologia BIM através de um enfoque interdisciplinar, sobretudo no que diz respeito às disciplinas de processo de construção.
\end{abstract}

Palavras-chave: BIM, Ensino, Interdisciplinaridade.

\begin{abstract}
BIM technology is increasingly being requested and used in the AEC market. Market dynamics and increasing complexity of generated products makes the use of compatible computational tools become increasingly important. However, follow and understand these advances has been a challenge for professional users of these systems. Therefore, many universities have courses to teach BIM to Architecture and Engineering students, which contributes to the consolidation of BIM as an important design tool. Nevertheless, many students still prefer to use the conventional CAD technology. This paper makes a survey of main difficulties faced by BIM students from Architecture and Urbanism course of UFJF and the reasons why most of these students will not use BIM technology in design process. This is an explanatory research that aims to understand the roots of difficulties in using BIM. For this, at first, a literature review was performed in order to raise the prior knowledge necessary to the design process in BIM platforms. Then, students chose the biggest barriers in using BIM. Results show that the greatest difficulty encountered by students is the large amount of data that must be entered into a BIM program, in order to design. According to the students, it slows down the process. Further analysis reveals, however, that this difficulty is mainly due to lack of knowledge on construction process itself, prior knowledge to design on BIM platforms. Hence, teaching the BIM technology to students through an
\end{abstract}


interdisciplinary approach, particularly with regard to the disciplines of the construction process, is more productive.

Keywords: BIM, Teaching, Interdisciplinarity.

\section{INTRODUÇÃO}

BIM é um conceito em desenvolvimento. Sendo assim, é difícil vinculá-lo a uma única definição. Como foi observado por Moreira ele vem sendo transformado "de acordo com as novas potencialidades da tecnologia e a própria habilidade dos usuários em manipulá-la e explorá-la" (MOREIRA, 2008, p. 67).

De uma forma geral, podemos dizer que BIM significa uma mudança entre apresentar a informação sobre a construção e representar essa informação. O BIM pode ser entendido também como uma filosofia de trabalho que integra profissionais das áreas de Arquitetura, Engenharia e Construção (AEC) na elaboração de um protótipo virtual único, que contenha todos os dados necessários à edificação de uma instalação. Assim, o que antes era fragmentado em diversas partes passa a ser incorporado e lido em uma plataforma única: projeto estrutural, arquitetônico, projetos complementares, orçamento, cálculo energético, gestão, etc. (MENEZES, 2011).

A tecnologia BIM surge, portanto, como uma saída para essa fragmentação, já que é uma plataforma para ser desenvolvida e gerenciada juntamente entre equipes de projeto e de construção. Fragmentação esta que além de estar presente nas ferramentas de trabalho também foi adotada na educação destes profissionais (FORGUES et al., 2011). Como afirmou Lawson (2006), a percepção do projeto sendo o resultado do ato criativo de apenas um indivíduo está enraizada no ensino de arquitetura e profundamente embutida no seu conhecimento prático e nas suas ferramentas.

Nesse contexto de fragmentação, a interoperabilidade e o gerenciamento de dados veem se tornando cada vez mais imprescindíveis ao processo de projeto de edificações. São exigências decorrentes da dinâmica inerente ao mercado de AEC e da complexidade cada vez maior dos produtos gerados, e que tornam essencial o uso de ferramentas computacionais compatíveis.

Sendo assim, a proposta do presente artigo é levantar as principais dificuldades encontradas pelos alunos da disciplina de BIM do curso de Arquitetura e Urbanismo da UFJF e as razões que levam grande parte destes a não adotar essa tecnologia no processo de projeto. Trata-se de uma pesquisa explicativa que visa compreender as raízes das dificuldades no uso dessa ferramenta. Num primeiro momento foi realizada uma revisão bibliográfica a fim de se levantar os conhecimentos prévios necessários ao desenvolvimento de projetos nas plataformas BIM. Depois os estudantes elencaram as maiores barreiras no uso dessa tecnologia e justificaram suas escolhas.

\section{REVISÃO BIBLIOGRÁFICA}

O BIM se constitui como uma mudança de paradigma sob a qual é repensada a forma com que o conhecimento em torno do projeto é construído pelos estudantes, profissionais e pesquisadores das áreas de AEC e afins (FORGUES et al., 2011). Tratase de uma tecnologia com uma variada gama de aplicações. No entanto, tal qual destacam Crespo \& Ruschel, "acompanhar e compreender estes avanços tem sido um desafio para os profissionais usuários destes sistemas" (CRESPO \& RUSCHEL, 2007, p. 3). 
De fato, os equívocos em torno do BIM são um dos principais motivos para o atraso na sua implementação (IBRAHIM, 2006). Uma das saídas para contornar esses equívocos é inserir o ensino do BIM nos currículos das universidades. Esse é um dos principais motivos do número de pesquisas sobre o ensino dessa tecnologia vir crescendo vigorosamente (AHN et al., 2013; EL-GAFY, 2006; FORGUES et al., 2011; SACKS E BARAK, 2009; WOO, 2006).

No entanto, a maioria das universidades ainda não atualizaram seus currículos e continuam ensinando apenas a tecnologia "CAD convencional". Isso se deve, por um lado, à novidade da tecnologia, já que poucas pessoas são capazes de ensiná-la e, por outro lado, ao dilema entre ensinar o programa e ensinar o próprio conceito da tecnologia por trás desse programa (IBRAHIM, 2007; FORGUES et al., 2011).

De uma forma geral, a falta de conhecimento das potencialidades de um programa é uma grande questão. Mesmo com softwares conhecidos com o AutoCad isso ocorre. Poucos profissionais são capazes de customizar esses programas, escrever macros, ou fazer uso de recursos mais "sofisticados". Com a tecnologia BIM essa questão se torna vital, pois são programas ainda mais difíceis de manipular (IBRAHIM, 2007).

Pesquisas apontam também que uma das principais razões para não usar a plataforma BIM é que os usuários não acham necessário mudar para essa tecnologia, pois acham que a tecnologia CAD convencional os atende bem (YAN E DAMIAN, 2008; SUERMANN, 2009).

Uma análise mais profunda, no entanto, indica que esta questão tem uma relação intrínseca com a formação desses profissionais. O desconhecimento dos benefícios provenientes da interoperabilidade da plataforma BIM, em muitos casos, reflete em uma atitude conservadora do ponto de vista tecnológico. A fragmentação das etapas de um projeto entre seus diversos participantes contribui para uma visão limitada do conjunto. Da mesma forma, a pesquisa e o ensino em Arquitetura e Engenharia também são fragmentados, devido à própria estrutura curricular das Faculdades.

Não por acaso, uma das primeiras iniciativas educacionais focadas na plataforma BIM e nas práticas integradas, desenvolvida na universidade de Stanford, em 1993, foi um curso denominado Computer Integrated Architecture, Engineering and Construction (Em tradução livre: Arquitetura, Engenharia e Construção integradas por Computador) (FORGUES et al., 2011).

A oposição entre fragmentação e integração parece estar no cerne dessa discussão. É evidente que um profissional da área de AEC será tanto melhor quanto mais conhecimento tiver das áreas relacionadas. Um arquiteto que tem certo conhecimento na área de engenharia e construção terá mais chances de conceber um bom projeto arquitetônico do que aquele que prescinde desse conhecimento. Da mesma forma, um engenheiro com conhecimentos da área de arquitetura está mais apto a desenvolver um bom projeto do que aquele que ignora esse saber.

Tendo isso em conta, buscamos investigar se a fragmentação presente no atual ensino da área de Arquitetura impacta negativamente os estudantes quando estes fazem uso de uma ferramenta que trabalha, sobretudo, com o conceito de integração.

\section{MÉTODO}

A fim de se descobrir a origem das dificuldades no uso da ferramenta BIM, realizamos uma pesquisa de caráter explicativo com os alunos da disciplina de BIM do departamento de Arquitetura e Urbanismo da Faculdade de Engenharia da UFJF. O 
objetivo principal desta pesquisa é verificar a hipótese de que a principal dificuldade dos estudantes no uso do BIM está na falta de conhecimento do processo de projeto como um todo.

Devido à complexidade da tecnologia BIM, optamos por não realizar entrevistas estruturadas. Apesar de ser mais eficiente na tabulação dos dados, a entrevista estruturada corre um risco maior de simplificação da questão, visto que ela oferece opções pré-determinadas. No universo da pesquisa explicativa, a entrevista não estruturada, por outro lado, corre um maior risco de induzir a erros, já que procura explicar o porquê de um fenômeno.

Sendo assim, foram realizadas entrevistas não estruturadas com 15 estudantes. Primeiro pedimos para o entrevistado eleger o maior obstáculo no aprendizado do BIM. Depois pedimos que justificasse sua escolha.

\section{RESULTADOS E DISCUSSÃO}

Como afirmado anteriormente, não é possível fazer uma compilação clara dos dados obtidos, dada a grande diversidade de dificuldades listadas . Iremos discutir, portanto, a mais citada, especialmente porque acreditamos ser a que mais contribui para a discussão colocada no presente artigo.

Os resultados mostram que a maior dificuldade encontrada pelos alunos é a grande quantidade de dados que precisam ser inseridos no programa para poder projetar, sendo apontada por quatro dos 15 estudantes entrevistados. Segundo eles, isso torna o processo mais lento e complexo.

Uma análise mais profunda revela, porém, que essa dificuldade deve-se principalmente à falta de conhecimento do próprio processo de construção, saber necessário à execução de projetos nas plataformas BIM. Ao justificar a maior barreira no uso do BIM, nove entrevistados, ou seja, $60 \%$ do total, afirmaram que não se sentiam capazes de finalizar um projeto completo fazendo uso dessa tecnologia, sobretudo porque não tinham um conhecimento suficiente do processo construtivo. Essa carência é reveladora. Como foi apontado por uma abrangente revisão bibliográfica, as três áreas mais importantes que um usuário de BIM deve conhecer são: tecnologia da informação, gestão e processo de construção (BARISON \& SANTOS, 2011).

Fica claro, para quem está se iniciando no uso do BIM, que essa tecnologia requer que se pense de uma forma diferente daquela da tecnologia CAD, para representar corretamente o desenho. Além da integração entre os diferentes profissionais, é desejável que estes tenham um conhecimento mais holístico sobre o processo de projeto. Muitos arquitetos já pensavam dessa maneira para projetar, mesmo usando a tecnologia CAD convencional (IBRAHIM, 2007).

Pesquisadores e professores já perceberam essa condição e muitas universidades já abriram disciplinas para o ensino do BIM. Mas o problema principal é que não basta apenas ensinar o BIM, mantendo a mesma compartimentada estrutura curricular. A indústria está se movendo rapidamente de práticas fragmentadas para práticas integradas através do IPD (Integrated Project Delivery). As fronteiras entre as especialidades estão desaparecendo, portanto, redefinindo a forma com que os projetos são desenvolvidos. 


\section{CONSIDERAÇÕES FINAIS}

BIM é uma mudança de paradigmas que nos leva a repensar em como o projeto e a construção são ensinados a arquitetos e engenheiros e também em como o conhecimento é gerado e transformado em novas formas de se organizar o trabalho.

O problema, nesse contexto de mudança de paradigmas, é que os estudantes estão adquirindo conhecimentos em projeto que podem tornar-se obsoletos em sua prática futura, e eles não estão preparados para trabalhar em ambientes digitais integrados (FORGUES et al., 2011).

Arquitetos, engenheiros e gestores estão entrando em um mundo complexo, que muda constantemente. Novas habilidades e competências tecnológicas são necessárias para dar conta dos desafios crescentes que eles irão encarar.

A mudança de ferramentas especializadas de projeto para esse modelo único e integrado desafia o próprio núcleo da prática profissional, da pesquisa e da educação. Ao se falar em BIM, há uma grande lacuna entre ensino e prática.

Portanto, introduzir complexas tecnologias configuracionais, como o BIM, compele profissionais do projeto e da construção a repensar o modo como trabalham e ajudam a gerar novos conhecimentos.

Contudo é importante ressaltar que para que esse quadro se concretize dentro do ideal de interoperabilidade, é necessário que esse processo se dê de forma integrada na grande área de AEC. Não basta que se mude o ensino para a Arquitetura, atualizando seus profissionais às novas ferramentas de simulação e projeto, se não houver também a mesma preocupação para o ensino da Engenharia. Mais uma vez trata-se do problema da fragmentação, que impede que o BIM seja explorado em toda a sua potencialidade, não só como ferramenta que auxilia o processo criativo e de projeto, mas também que colabora no planejamento e gestão de todo o processo de desenvolvimento da edificação.

\section{REFERÊNCIAS}

AHN, Yong Han; LEE, Namhun ; CHO, Chung-suk. Building Information Modeling: Systematic Course Development for Undergraduate Construction Studentes. Journal of Professional Issues in Engineering Education and Practice, pp.130411195411006, 2013.

BARISON, M.B.; SANTOS, E.T. The competencies of BIM specialists: a comparative analysis of the literature review and job ad descriptions. In: Computing in Civil Engineering, $594-602,2011$.

CRESPO, Cláudia Campos; RUSCHEL, Regina Coeli. Ferramentas BIM: um desafio para a melhoria no ciclo de vida do projeto. III Encontro de Tecnologia de Informação e Comunicação na Construção Civil, Porto Alegre, 11 e 12 de julho, 2007. Disponível em: < http://noriegec.cpgec.ufrgs.br/tic2007/artigos/A1085.pdf>. Acesso em 06 mai. 2013.

EL-GAFY, M. Proceeding of the 2009 ASC Region III Conference, 2006. 
FORGUES, Daniel; STAUB-FRENCH, Sheryl; FARAH, Leila M. Teaching building design and construction engineering. Are we ready for the paradigma shift? Proceedings of the 2nd Annual CEEA Conference: Memorial University St. John's, Newfoundland June 6-8, 2011.

IBRAHIM, Magdy M. To BIM or not to BIM, This is NOT the Question - How to Implement BIM Solutions in Large Design Firm Environments. Communicating Space(s) [24th eCAADe Conference Proceedings], Volos (Greece), September. Pp. 262267, 2006.

IBRAHIM, Magdy M. Teaching BIM, what is missing?. 3rd Int "'l ASCAAD Conference on Em,,body ${ }^{e e}$ ing Virtual Architecture [ASCAAD-07, Alexandria, Egypt], 2007.

LAWSON, B. How Designers Think: The Design Process Demystified. Oxford: Architectural Press, 2006.

MENEZES, Gilda Lúcia B B. Breve histórico de implantação da plataforma BIM. Cadernos de Arquitetura e Urbanismo, v.18, n.22, $21^{\circ}$ sem. 2011.

MOREIRA, Thomaz Passos Ferraz. Universidade de Brasília. A influência da parametrização dos softwares CADD arquiteturais no processo de projetação arquitetônica. 2008. 165p. Dissertação (Mestrado). Universidade de Brasília.

SACKS, R.; BARAK, R. Teaching building information modeling as an integral part of freshman year civil engineering education. Journal of Professional Issues in Engineering Education and Practice, 136(1), p. 30-38, 2009.

SUERMANN, Patrick C. Evaluating the impact of building information modeling (BIM) on construction. 2009. 230p. Tese (Doutorado). University of Florida.

WOO, J. H. BIM (Building information modeling) and pedagogical challenges. Proceedings of the 43rd ASC National Annual Conference (pp. 12-14), 2006.

YAN, H.; DAMIAN, P. Benefits and barriers of building information modelling. 12th International Conference on Computing in Civil and Building Engineering, 2008. 